\title{
Incidencia de cáncer en niños chilenos infectados por VIH
}

\author{
Julia Villarroel, Ana M. Álvarez, Ana Chávez, José Cofré, M. Isabel Galaz, \\ Patricio Ledesma, Anamaría Peña, Eloisa Vizueta y Elba Wu
}

Comité Nacional de SIDA Pediátrico.

No hubo fuentes de financiamiento. No hubo conflicto de interés de los autores.

Recibido: 19 de diciembre de 2014 Aceptado: 8 de octubre de 2015

Correspondencia a: Julia Villarroel Barrera julia.villarroel@vtr.ne

\section{Incidence of cancer in Chilean HIV-infected children}

Background: Pediatric HIV (+) patients have a 100 times greater risk of cancer than HIV (-) children. Objective: To describe in Chilean HIV $(+)$ children, cancer types, its appearance in relation to the stages of HIV disease and mortality. Methods: A protocol was created to know some characteristics of these patients from the point of view of their HIV infection and cancer pathology. Results: Of 360 HIV (+) children confirmed by the Institute of Public Health to May 2014, 9 patients with neoplastic disease (2.5\%) were diagnosed. All the children were on ART, had more than three years of evolution of HIV infection and were in moderate to severe clinical/immunological stages. Lymphoma was the most common cancer. Five children, has received therapy according to Programa Infantil Nacional de Drogas Antineoplásicas (PINDA). There was no interaction between cancer treatment and antiretroviral therapy. Mortality was 13.8 x 1000 (5 cases). Conclusions: The incidence and type of neoplasia is consistent with the international literature, with less survival than HIV $(+)$ children without tumors. The occurrence of cancer was observed in children with moderate to severe clinical and immunological compromise.

Key words: Malignancies, HIV, non-Hodgkin's lymphoma.

Palabras clave: Manifestaciones tumorales, VIH, linfoma no Hodgkin.

\section{Introducción}

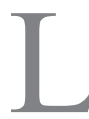
os niños con infección por el virus de la inmunodeficiencia humana (VIH) tienen alto riesgo de desarrollar neoplasias ${ }^{1}$. Los primeros casos pediátricos fueron reportados en paciente hemofílicos, en muchos de los cuales la aparición de cáncer $(\mathrm{Ca})$ fue la primera manifestación de infección por $\mathrm{VIH}^{2}$. Aunque la prevalencia de $\mathrm{Ca}$ es menor en los niños que en los adultos infectados con VIH, se ha estimado que los pacientes pediátricos tienen un riesgo 100 veces mayor de presentar Ca que los niños no infectados con VIH. Estudios extranjeros muestran una incidencia de tumores malignos en niños infectados con VIH de 1 a $2 \%$. Los cánceres más frecuentes son linfomas, especialmente no Hodgkin, sarcomas y tumores del músculo liso como leiomiosarcoma.

\section{Objetivos}

Conocer la incidencia de cánceres en los niños con infección por VIH controlados por el Comité Nacional de SIDA Pediátrico. Describir los tipos de Ca, la aparición en relación a las etapas de la enfermedad por VIH y la letalidad. Evaluar la incidencia de neoplasias antes del inicio de la terapia antirretroviral (TARV) y post terapia.

\section{Material y Métodos}

Se revisó la base de datos nacionales seleccionando los niños que presentaban alguna enfermedad neoplásica y se creó un protocolo con datos de edad, sexo, etapa evolutiva en el diagnóstico de infección por VIH, TARV recibida, tiempo de evolución entre el diagnóstico de infección por VIH y la detección del $\mathrm{Ca}$, etapa en que se encontraba al diagnóstico de $\mathrm{Ca}$, terapias antineoplásicas usadas, interacciones entre la TARV y la terapia anti-neoplásica, tipo y localización de los cánceres y mortalidad.

\section{Resultados}

De un total de 360 niños con infección por VIH controlados por el Comité desde 1987 a mayo de 2014, se encontraron nueve pacientes con alguna neoplasia, con una incidencia acumulada de $2,5 \%$. La forma de adquisición de la infección por VIH en estos pacientes fue por transmisión vertical (TV), las edades al diagnóstico de estar infectado con VIH fluctuaron entre un mes y 47 meses (promedio 17,8 meses). Cinco pacientes eran de sexo masculino $(55,5 \%)$.

Al momento del diagnóstico de infección por $\mathrm{VIH}$, ocho de los nueve niños se encontraban en etapa no SIDA. El tiempo entre el diagnóstico de infección por VIH y la detección de la neoplasia varió entre 39 y 180 meses (promedio 79,8 meses).

Las etapas clínicas e inmunológicas de la infección por $\mathrm{VIH}$ al diagnóstico de Ca fluctuaron entre $\mathrm{B} 1$ a $\mathrm{C} 3$, ocho niños estaban en etapa de SIDA. El porcentaje de linfocitos T (LT) CD4 varió entre 4 y 39\% y sólo cuatro pacientes tenían cargas virales $(\mathrm{CV})$ indetectables.

Se pesquisaron cuatro linfomas, de los cuales dos fueron linfomas de Burkitt, un linfoma de células $\mathrm{T}$ y un linfoma no Hodgkin. Los restantes cánceres fueron dos sarcomas, un teratoma, una leucemia linfoblástica aguda 


\begin{tabular}{|c|c|c|c|c|c|c|c|c|c|}
\hline & Caso 1 & Caso 2 & Caso 3 & Caso 4 & Caso 5 & Caso 6 & Caso 7 & Caso 8 & Caso 9 \\
\hline Sexo & M & $\mathrm{F}$ & M & $\mathrm{F}$ & $\mathrm{F}$ & M & M & $\mathrm{F}$ & M \\
\hline Región & $\mathrm{RM}$ & RM & RM & RM & RM & Arica & RM & RM & RM \\
\hline Mecanismo transmisión & Vertical & Vertical & Vertical & Vertical & Vertical & Vertical & Vertical & Vertical & Vertical \\
\hline Año de diagnóstico de la infección por VIH & 1993 & 1996 & 1997 & 1997 & 1999 & 2001 & 2003 & 2003 & 2004 \\
\hline Edad al diagnóstico de la infección por VIH & $13 \mathrm{~m}$ & $4 \mathrm{~m}$ & $36 \mathrm{~m}$ & $40 \mathrm{~m}$ & $4 \mathrm{~m}$ & $6 \mathrm{~m}$ & $10 \mathrm{~m}$ & $1 \mathrm{~m}$ & $47 \mathrm{~m}$ \\
\hline Etapa clínico/inmunológica al diagnóstico de infección por VIH & B1 & B2 & A1 & B1 & N1 & C3 & C2 & N1 & B2 \\
\hline Tipo de tumor & Teratoma & L pleomórfico & L Burkitt & S indiferenciado & $\mathrm{S}$ alto grado & L Burkitt & Tu fosa posterior & LLA & L Hodgkin \\
\hline Etapa clínica al diagnóstico de tumor & B & B & B & B & C & c & c & c & C \\
\hline Etapa inmunológica al diagnóstico de tumor & 1 & 2 & 3 & 3 & 3 & 3 & 1 & 3 & 1 \\
\hline $\begin{array}{l}\text { Tiempo entre diagnóstico de la infección por VIH y } \\
\text { diagnóstico de CA }\end{array}$ & $15 \mathrm{a}$ & 3 a $2 \mathrm{~m}$ & 3 a $10 \mathrm{~m}$ & 9 a & 8 a 3 m & 3 a $5 \mathrm{~m}$ & 8 a $10 \mathrm{~m}$ & $7 a$ & 4 a $1 \mathrm{~m}$ \\
\hline Años de TARV al diagnóstico de CA & $15 \mathrm{a}$ & 2 a $2 \mathrm{~m}$ & 1 a $9 \mathrm{~m}$ & 8 a $3 \mathrm{~m}$ & 9 a $1 \mathrm{~m}$ & 3 a $2 \mathrm{~m}$ & 9 a & $8 \mathrm{~m}$ & 3 a $7 \mathrm{~m}$ \\
\hline
\end{tabular}

\begin{tabular}{|c|c|c|c|c|c|c|c|c|c|}
\hline Características & Caso 1 & Caso 2 & Caso 3 & Caso 4 & Caso 5 & Caso 6 & Caso 7 & Caso 8 & Caso 9 \\
\hline Tipo de tumor & Teratoma & $\begin{array}{l}\text { Linfoma } \\
\text { células T }\end{array}$ & $\begin{array}{l}\text { Linfoma } \\
\text { Burkitt }\end{array}$ & $\begin{array}{c}\text { Sarcoma } \\
\text { indiferenciado }\end{array}$ & $\begin{array}{l}\text { Sarcoma alto } \\
\text { grado }\end{array}$ & $\begin{array}{l}\text { Linfoma } \\
\text { Burkitt }\end{array}$ & $\begin{array}{l}\text { Tumor fosa } \\
\text { posterior }\end{array}$ & LLA & $\begin{array}{l}\text { Linfoma } \\
\text { Hodgkin }\end{array}$ \\
\hline $\begin{array}{l}\text { Localización } \\
\text { tumor }\end{array}$ & Testicular & Mediastino & $\begin{array}{l}\text { Recto- } \\
\text { sigmoideo }\end{array}$ & Retrofaríngeo & Endobronquial & Desconocido & Cerebro & Sangre & Mediastino \\
\hline $\begin{array}{l}\text { Clasificación } \\
\text { cáncer }\end{array}$ & Embrionario & $\begin{array}{l}\text { Pleomórfico } \\
\text { de alto grado }\end{array}$ & Etapa III & $\begin{array}{l}\text { Estadio IV, } \\
\text { grupo IV }\end{array}$ & $\begin{array}{l}\text { Alto grado } \\
\text { metastásico }\end{array}$ & $\begin{array}{l}\text { Etapa II, } \\
\text { riesgo } 2\end{array}$ & Desconocido & $\begin{array}{c}\text { Estirpe B } \\
\text { madura }\end{array}$ & Grupo 3 \\
\hline $\begin{array}{l}\text { Terapia } \\
\text { anticancerosa }\end{array}$ & Sí & No & Sí & Sí & No & Sí & No & Sí & Sí \\
\hline Vivo o muerto & Vivo & Muerto & Vivo & Muerto & Muerto & Vivo & Muerto & Muerto & Vivo \\
\hline $\begin{array}{l}\text { Causa de } \\
\text { muerte }\end{array}$ & - & $\begin{array}{l}\text { Infección por } \\
\qquad M A C\end{array}$ & - & $\begin{array}{l}\text { Septicemia por Pseu- } \\
\text { domonas aeruginosa }\end{array}$ & $\begin{array}{c}\text { Hemorragia } \\
\text { pulmonar masiva }\end{array}$ & - & $\begin{array}{l}\text { Enclava- } \\
\text { miento }\end{array}$ & $\begin{array}{l}\text { Infección } \\
\text { por ABA }\end{array}$ & - \\
\hline $\begin{array}{l}\text { Tiempo de } \\
\text { sobrevida }\end{array}$ & 96 meses & 15 días & 98 meses & 15 meses & 1 mes & 108 meses & 3 días & 6 días & 74 meses \\
\hline
\end{tabular}

y un tumor cerebral que no fue posible identificar por el precoz fallecimiento del niño (Tabla 1).

Cinco pacientes recibieron terapia oncológica de acuerdo al protocolo del Programa Infantil Nacional de Drogas Antineoplásicas (PINDA). En los otros cuatro la evolución fue muy rápida y no se alcanzó a iniciar tratamiento. No se detectó interacciones farmacológicas entre la terapia antineoplásica y la TARV.

Todos los pacientes se encontraban en tratamiento con TARV en un período de tiempo que fluctuó entre 1 año 9 meses y 15 años, estando la infección por VIH en etapa clínica e inmunológica moderada a grave. Todos tenían una evolución de la infección por VIH mayor a 3 años al momento de presentar la neoplasia.

Las complicaciones observadas en los niños con Ca y quimioterapia, fueron de causa infecciosa, preferentemente neutropenias febriles, infección por citomegalovirus (CMV) y septicemias por Pseudomonas aeruginosa.
La mortalidad de la serie fue 55,5\% (5 casos). La sobrevida global posterior a la neoplasia, fluctuó entre 3 días y 163 meses. Las causas de muerte en tres niños fueron infección por $P$. aeruginosa (n: 1); en los otros dos niños, fueron enclavamiento debido a la localización del tumor (n: 1) y hemorragia pulmonar masiva (n: 1).

Cuatro de los cinco niños fallecidos no recibieron tratamiento oncológico, ya que su sobrevida fue entre tres y 38 días, todos se encontraban en etapas muy avanzadas de la neoplasia o adquirieron infección por agentes multiresistentes, lo que motivó su deceso. El único caso que recibió quimioterapia tuvo una sobrevida de 15 meses y la causa de muerte también fue de origen infeccioso.

\section{Discusión}

Cada año se diagnostican alrededor de 130 casos de cáncer por millón de niños sin infección por VIH (inci- 
dencia de $0,13 \%$ a 0,15 por 1.000$)^{3}$. Estudios efectuados en Estados Unidos de América (E.U.A.) y en Europa en los niños infectados por el VIH, refieren incidencias que fluctúan entre 9,4 por 1.000 a 20 x 1.000, previas al inicio de la TARV ${ }^{4}$

Con el inicio de la TARV se logró una mejor calidad de vida y mayor sobrevida, pero esta sobrevida se ha visto acompañada de un alto riesgo de desarrollar malignidades no definitorias de SIDA, aunque los cánceres indicadores de SIDA han disminuido considerablemente ${ }^{5}$. Esto ha sido demostrado en estudios extranjeros como uno efectuado en Italia, en el que se observó una disminución importante de los cánceres en niños con infección por VIH adquirida en el período perinatal, de 4,49 por 1.000 personas por año a $0,76 \times 1.000$ personas por año; no obstante, la incidencia de Ca continua elevada, cinco veces mayor que para la población no infectada por $\mathrm{VIH}^{6}$.

En nuestro estudio todos los casos se presentaron entre el año 1999 y el 2012, ocurriendo después del inicio de la triterapia. Probablemente esto se explica por la mayor sobrevida alcanzada por nuestros pacientes después del inicio de la TARV.

Otros estudios comparando la incidencia de cánceres ocurridos en la edad pediátrica entre la era pre-TARV y la post TARV, demostraron una disminución en $87 \%$ de sarcoma de Kaposi y $60 \%$ de linfoma no Hodgkin, ya que estos tumores se asocian con un recuento bajo de LT CD47 . Sin embargo, se mantiene alta la incidencia de cánceres no definitorios de SIDA, los cuales se asocian a co-infecciones con otros virus como Epstein-Barr (VEB) es el caso de linfoma de Burkitt, linfoma de Hodgkin y leiomiosarcoma ${ }^{8}$. Además se aprecian cambios en la presentación de esas malignidades ubicándose en sitios inusuales y ocurriendo en pacientes con recuentos de LT CD4 moderadamente disminuidos.

En los casos estudiados por nosotros, el porcentaje de LT CD4 en los pacientes que presentaron alguna neoplasia fluctuó entre 4 y 39\%, encontrándose en su mayoría en etapa clínica de SIDA y/o inmunológica de inmunosupresión grave.

Las causas por las cuales los niños infectados por VIH tienen alto riesgo de desarrollar alguna malignidad son diversas; se relacionan con una alteración de su sistema inmune, debido a una depleción progresiva de LT CD4, con pérdida de las funciones inmunes y además hay una compleja interacción con otros virus oncogénicos como $\mathrm{VEB}$, virus herpes tipo $8, \mathrm{CMV}$ y también con virus papiloma ${ }^{9,10}$.

Los pacientes con infección por VIH presentan manifestaciones de activación crónica del sistema inmune lo que se evidencia por altos niveles de interleuquinas pro-inflamatorias (IL-6, IL-10, FNT, interferón alfa, etc.), activación policlonal de las células $\mathrm{B}$ y un acelerado envejecimiento del sistema inmune ${ }^{11}$. Por otra parte, la infección por VIH causa precozmente una masiva depleción de LT CD4 a nivel intestinal; esto lleva a una brecha en la mucosa intestinal con la subsecuente translocación de productos bacterianos como lipopolisacáridos y segmentos de ADN de las células muertas. Estos productos ingresan a la circulación, son reconocidos por receptores y esa unión genera gran liberación de citoquinas pro-inflamatorias ${ }^{12,13}$.

La TARV ha permitido elevar el recuento de LT CD4; sin embargo, la desregulación del sistema inmune persiste en muchos niños a pesar de una terapia adecuada, incluso con recuentos normales de LT CD4.

Las alteraciones inmunes que se producen en los niños infectados por el VIH representan un escenario especial para la co-infección con otros virus, tales como, el VEB. Se ha demostrado que las células infectadas por VEB escapan del control inmunológico y, por otra parte, la inmunosupresión promueve la emergencia de clones co-infectados transformados, que no son reconocidos ni eliminados por el sistema inmune, ya que se ha visto que la infección VIH se asocia con la pérdida selectiva de LT CD4 específicos para $\mathrm{VEB}^{14,15}$.

Estudios demuestran ${ }^{16,17}$ que un ascenso en el recuento de LT CD4, pero sin supresión viral, se acompaña de un aumento de la CV de virus de VEB y también un aumento de las concentraciones plasmáticas de inmunoglobulinas. Este es un aspecto importante, ya que 20 a $30 \%$ de los niños con infección por VIH desarrollan una respuesta discordante a la TARV, en la cual se logran altos niveles de LT CD4, pero con CV persistentes para el virus VIH. Cargas elevadas de VEB conllevan a altos niveles de citoquinas pro-inflamatorias lo que aumenta la traslocación bacteriana en el tubo digestivo y perpetúa el fenómeno inflamatorio incidiendo en la persistencia de cánceres en la era TARV. Sólo 4 (44\%) de nuestros pacientes con algún proceso oncológico, presentaban $\mathrm{CV}$ indetectables.

La activación policlonal de las células $\mathrm{B}$, independiente del recuento de $\mathrm{LT}$ CD4, favorece la expansión de las células B infectadas y esto también promueve el inicio de los cánceres.

Otro aspecto importante en la patogenia de los cánceres, tanto en adultos como en niños, es que los sujetos infectados por VIH tienen cambios semejantes a los ancianos en su sistema inmune, dado por una reducción de la función del timo, descenso de los LT CD28, molécula fundamental para la activación de las células T. Este envejecimiento se ve también en los niños, incluso al año de vida. Lo anterior apoya la importancia de mantener la CV indetectable, evitando la senescencia del sistema inmune y la disfunción de los LT CD8 y CD28. Situación que es más marcada en los niños infectados por TV. La acumulación progresiva de LT senescentes facilita también el desarrollo de cánceres ${ }^{18,19}$.

También se ha visto que los fármacos usados en la TARV podrían causar un envejecimiento precoz. Los inhibidores nucleósidos de la transcriptasa reversa (INTR), inhiben la telomerasa reversa, enzima fundamental en la expansión de los linfocitos, así como en la regulación de la longitud de los telómeros. Zidovudina (AZT), didanosina 
(ddI) y abacavir (ABC) inhiben la telomerasa in vitro, generando un acortamiento de los telómeros ${ }^{20}$. En adultos se ha demostrado que la duración de la TARV con INTR provoca acortamiento de los telómeros y esto contribuye al envejecimiento precoz ${ }^{21}$. En niños no se ha probado; sin embargo, sí se encontró relación entre la viremia persistente y el acortamiento de éstos. El acortamiento de los telómeros lleva a inestabilidad genética, lo cual es la llave para el inicio de procesos oncogénicos ${ }^{22}$. Nuestros pacientes tuvieron uno o dos esquemas de TARV en las que siempre hubo INTR.

Estudios efectuados en E.U.A. ${ }^{23}$ y en Italia ${ }^{24}$, muestran que los linfomas, especialmente los de células B y los primarios del sistema nervioso central, son los principales cánceres vistos en los niños infectados por VIH, lo que contrasta fuertemente con el espectro de malignidades que ocurren en niños sin infección por VIH, donde las patologías oncológicas más frecuente son leucemias (40\%), seguido por tumores del sistema nervioso central $(17 \%)$ y linfomas $(13 \%)^{25}$. El tipo de Ca que aparece con mayor frecuencia en los niños estudiados por nosotros es el linfoma, al igual que lo publicado para países desarrollados. Estos tumores aparecen incluso con sistema inmune conservado. Los niños con linfomas en nuestra serie, estaban en etapa clínica-inmunológica que fluctuó entre compromiso moderado y grave (B2 a C3). Los linfomas de Burkitt, así como el linfoma de Hodgkin, se relacionan con la co-infección por VEB. Un estudio efectuado en Texas permitió evidenciar los factores de riesgo para presentar Ca y se demostró que la presencia de una $\mathrm{CV}$ para $\mathrm{VEB}>50$ copias $\times 10^{5}$ células mononucleares, se asoció fuertemente con riesgo de $\mathrm{Ca}$ en los niños con infección por VIH con recuentos de CD4 > 200 céls $/ \mathrm{ml}$. No se observó en este estudio, que la terapia con AZT confiriera protección, tampoco la ruta de infección VIH se asoció con mayor riesgo de cáncer ${ }^{26}$. Desgraciadamente en nuestros pacientes no se efectuó estudio para VEB.

Los sarcomas, otros tumores vistos en el estudio (2 casos) aparecen como segunda frecuencia de cánceres en estos pacientes. Sarcomas diferentes a Kaposi se ven en estos pacientes, excepto en África sub-sahariana donde el sarcoma de Kaposi ha aumentado en forma considerable especialmente en niños, superando el $20 \%$; esta mayor frecuencia está relacionada con la co-infección por virus herpes $8^{27-29}$. Otros cánceres vistos en niños con manifestaciones no definitorias de SIDA son el tumor de Willms, las leucemias y otros tipos como teratomas, los que presentan histología inusual. Esto se evidenció en nuestros casos donde tuvimos una leucemia linfoblástica aguda y un teratoma de localización testicular.

Un aspecto interesante de la mayor incidencia de cánceres en niños con infección por VIH, ha sido ver si los niños expuestos en la etapa perinatal a TARV pueden tener mayor incidencia de neoplasias. Un estudio inglés ${ }^{30}$ no demostró diferencias con la población normal en la incidencia de cánceres en los niños que recibieron TARV con AZT. No obstante, el riesgo relativo para los niños expuestos a la combinación de ddI y lamivudina (3TC) fue mayor. Lo anterior plantea otra línea de estudio. Nuestros pacientes se infectaron por TV de la infección por VIH; sin embargo, ninguno fue sometido a protocolo de prevención de TV.

La mortalidad por $\mathrm{Ca}$ en pacientes bajo 15 años de edad ha disminuido en Chile gracias a los grandes avances en protocolos de tratamientos enmarcados dentro del PINDA: en general es de 3,4 x 100.000 niños. También se ha producido un descenso en la mortalidad en los linfomas y sarcomas de hasta $44 \%{ }^{31}$. Nosotros tuvimos una mortalidad elevada, que se produjo por sobreinfecciones $\mathrm{y}$ en un caso por enclavamiento debido a la localización del tumor; esto difiere de otras publicaciones en las cuales la mortalidad se produjo por toxicidad a las terapias (triterapia y terapia oncológica). Probablemente nuestros pacientes, al estar en etapas avanzadas de la enfermedad por VIH, tenían un sistema inmunológico muy disminuido que originó una mayor propensión a las infecciones ${ }^{32}$. Sin embargo, las cifras son semejantes e incluso algo menores a las publicadas en estudios internacionales; probablemente estas cifras aumenten al incrementarse el número de casos de $\mathrm{Ca}$.

\section{Conclusiones}

La sobrevida de los pacientes bajo 15 años de edad, no infectados con $\mathrm{VIH}$, con $\mathrm{Ca}$, ha mejorado considerablemente, fluctuando entre 73 y $98 \%$ a 5 años, dependiendo del tipo de Ca. La mortalidad asociada a neutropenia febril post-quimioterapia de alto riesgo fluctúa entre 2 y $3 \% \%^{33-35}$. Se ha demostrado que los niños con Ca e infección por VIH tienen una sobrevida menor. En nuestro estudio, la sobrevida global ha sido en promedio de 4,8 años. No obstante, la sobrevida en los niños que fallecieron fue muy breve, ya que se encontraban en etapas avanzadas del Ca así como de la infección por VIH y por otra parte, no alcanzaron a recibir terapia oncológica.

\section{Resumen}

Introducción: Los pacientes pediátricos con infección por VIH tienen un riesgo 100 veces mayor de presentar cáncer que los niños no infectados. Objetivos: Describir en niños chilenos con infección por VIH, los tipos de cáncer, su aparición en relación a las etapas de la enfermedad por VIH y la letalidad. Material y Métodos: Se creó un protocolo para conocer algunas características de estos pacientes desde el punto de vista de su infección por VIH y su patología oncológica. Resultados: De 360 niños infectados confirmados por el Instituto de Salud Pública a mayo de 2014, se diagnosticaron nueve casos con patología oncológica $(2,5 \%)$. Todos los niños estaban con TARV, tenían una evolución de infección por VIH 
mayor a 3 años, en etapas clínicas/inmunológicas moderada a grave. Linfoma fue el cáncer más frecuente. Cinco niños, recibieron terapia de acuerdo al Programa Infantil Nacional de Drogas Antineoplásicas (PINDA). No hubo interacción entre tratamiento anti-neoplásico y terapia anti-retroviral. La mortalidad fue de $13,8 \times 1.000$ (5 casos). Conclusiones: La incidencia y tipo de neoplasias está de acuerdo con lo comunicado en la literatura científica internacional, con sobrevida inferior a los niños con infección por VIH sin neoplasias. La aparición de cáncer se observó en niños con larga evolución y compromiso clínico e inmunológico moderado a grave.

\section{Referencias bibliográficas}

1.- Mueller B. Cancers in children infected with the human immunodeficiency virus. Oncology 1999; 4: 309-17.

2.- Rabkin C, Hilgartner M, Hedberg K. Incidence of lymphomas and other cancers in HIV-infected and HIV-uninfected patients with hemophilia. JAMA 1992; 267: 1090-4.

3.- Stack M, Walsh P M, Comber H. Childhood cancer in Ireland: a population-based study. Arch Dis Childhood 2007; 92: 890-7.

4.- Biggar R, Frisch M, Goedert J. Risk of cancer in children with AIDS. JAMA 2000; 284: 205-9.

5.- Álvaro-Meca A, Micheluod D, Jensen J, Díaz A, García-Álvarez M, Resino S. Epidemiologic trends of cancer diagnoses among HIV-infected children in Spain From 1997-2008. Pediatr Infect Dis J 2011; 30: 764-8.

6.- Chiappini E, Galli L, Tovo P. Cancer rates after year 2000 significantly decrease in children with perinatal HIV infection: A study by the Italian register for HIV infection in children. J Clin Oncol 2007; 25: 97-101.

7.- $\quad$ Simard E, Shields M, Bhatia K, Engels E. Long-term cancer risk among people diagnosed with AIDS during childhood. Cancer Epidemiol Biomarkers Prev 2012; 21: 148-54.

8.- Bhatia K, Shiels M, Berg A, Engels E. Sarcomas other than Kaposi sarcoma occurring in immunodeficiency: interpretations from a systematic literature review. Curr Opin Oncol 2012; 24: 537-46.

9.- Mc Clain K, Leach C, Jenson H. Association of Epstein-Barr virus with leiomyosarcomas in children with AIDS. N Engl J Med 1995; 332: 12-8.

10.- Chiappini E, Berti E, Gianesin K, Petrara M R, Galli L, Giaquinto C, et al. Pediatric human immunodeficiency virus infection and cancer in the highly active antiretroviral treatment (HAART) era. Cancer Letters 2014; 347 (1): 38-45.

11.- Breen E, Hussain S, Magpantay L, Jakobson L, Detels R, Rabkin C, et al. B-cell stimulatory cytokines and markers of immune activation are elevated several years prior to the diagnosis of systemic AIDS associated non-Hodgkin B cell lymphoma. Cancer Epidemiol. Biomarkers Prev 2011; 20: 1303-14

12.- Brenchley J, Price D, Scharker T, Asher T, Silvestri G, Rao S, et al. Microbial translocation is a cause of systemic immune activation in chronic HIV infection. Nat Med 2006; 12: 136571.

13.- Marks M, Rabkin C, Engels E, Bush E, Kopp W, Rager H, et al. Markers of microbial translocations and risk of AIDS-related lymphoma. AIDS 2013; 27: 469-74.

14.- Münz C, Moormann A. Immune escape by Epstein-Barr virus associated malignancies. Semin Cancer Biol 2008; 18: 381-7.

15.- Piriou E, van Dort K, Nanlohy M, van Oers F, Miedema F, van Baarle D. Loss of EBNA 1-specific memory CD4+ and CD8+ T cells in HIV-infected patients progressing to AIDSrelated non Hodgkin lymphoma. Blood 2005; 106: 3166-74.

16.- Petrara M, Cattelan A, Zanchetta M, Sasset L, Freguja R, Gianesin K, et al. Epstein-Barr virus load and immune activation in human immunodeficiency type 1-infected patients. J Clin Virol 2012; 53: 195-200.

17.- Righetti E, Ballon G, Ometto L, Cattelan A, Menin C, Zanchetta M, et al. Dynamics of Epstein-Barr virus in human immunodeficiency virus type-1 infected subjects on highly active antiretroviral therapy. AIDS 2002; 16: 63-73.

18.- Dessai S, Landay A. Early immune senescence in HIV disease. Curr HIV/AIDS Rep 2010; 7: 4-10.

19.- Mansoor N, Abel B, Scriba T, Hughes J, Kock M, Tameris M, et al. Significantly skewed memory CD8+ T cell subsets in HIV-1 infected infants during the first year of life. Clin Immunol 2009; 130: 280-9.

20.- Tressler R, Godfrey C. NRTI backbone in HIV treatment: will it remain relevant. Drugs 2012; 72: 2051-62.

21.- Leeansyah E, Cameron P, Solomon A, Tennakoon S, Velayudham P, Gouillou M, et al. Inhibition of telomerasa activity by human immunodeficiency virus (HIV) nucleos(t)ide reverse transcriptase inhibitors: a potential factor contributing to HIV-associated accelerated aging. J Infect Dis 2013; 207: 1157-65.

22.- Artandi S, DePhino R. Telomeres and telomerase in cancer. Carcinogenesis 2010; 31: 9-18.

23.- Biggar R, Frich M, Goedert J. Risk of cancer in children with AIDS. JAMA 2000; 2: 205-9.

24.- Caselli D, Klersy C, de Martino M. Human immunodeficiency virus-related cancer in children: incidence and treatment outcomereport of the Italian register. J Clin Oncol 2000; 18: 3854-61.

25.- Vargas L. Cáncer en pediatría: Aspectos generales. Rev Chil Pediatr 2000; 71: 283-95.

26.- Pollock B, Jenson H, Leach C, McClain K, Hutchinson R, Garzarella L, et al. Rish factors for pediatric human immunodeficiency virusrelated malignancy. JAMA 2003; 289: 2393-9.

27.- Gantt S, Kakuru A, Wald A, Walusansa V, Corey L, Casper C, et al. Clinical presentation and outcome of epidemic Kaposi sarcoma in Ugandan children. Pediatr Blood Cancer 2010; 54: 670-4.

28.- Davidson A, Wainwright R, Stone D, Kruger M, Hendricks M, Geel J, et al. Malignancies in South African Children with HIV. J Pediatr Hematol Oncol 2014; 36: 111-7.

29.- Sinfield R, Molyneux E, Banda K, Borgstein E, Broadhead R, Hesseling P. Spectrum and presentation of pediatric malignancies in the HIV era: experience from Blantyre, Malawi, 1998-2003. Pediatr Blood Cancer 2007; 48: 515-20.

30.- Benhammou V, Warszawski J, Bellec S, Doz F, André N, Lacour B, et al. Incidence of cancer in children perinatally exposed to nucleoside reverse transcriptase inhibitors. AIDS 2008; 22: 2165-77.

31.- Cerda J, Romero M, Wietstruck M. Mortalidad por cáncer infantil en Chile. Modelo de transición epidemiológica en la infancia. Rev Chil Pediatr 2008; 79: 481-7.

32.- Stefan D, Stone D. Children with cancer and HIV infection: What is different about them? J Pediatr Hematol Oncol 2013; 8: 590-6.

33.- Santolaya M E, Álvarez A, Becker A, Avilés C L, Becker A, Mosso C. Admission clinical and laboratory factors associated with death in children with cancer during a febrile neutropenia episode. Pediatr Infect Dis J 2007; 26: 794-8.

34.- Basu S K, Fernández I D, Fisher S G, Asselin B L, Lyman G H. Length of stay and mortality associated with febrile neutropenia among children with cancer. J Clin Oncol 2005; 23: 7958-66.

35.- Paganini H, Aguirre C, Puppa G, Garbini C, Javier R G, Ensinck G. A prospective, multicentric screening system to predict mortality in febrile neutropenic children with cancer. Cancer 2007; 109: 2572-9. 